\title{
Theoretical and Experimental Studies on Alkali Metal Phenoxyacetates
}

\author{
E. Regulska, M. Samsonowicz, R. Świsłocka, and W. Lewandowski \\ Division of Chemistry, Bialystok University of Technology, 15-435 Bialystok, Poland \\ Correspondence should be addressed to E. Regulska, e.regulska@pb.edu.pl
}

Copyright (C) 2012 E. Regulska et al. This is an open access article distributed under the Creative Commons Attribution License, which permits unrestricted use, distribution, and reproduction in any medium, provided the original work is properly cited.

\begin{abstract}
Optimized geometrical structures of alkali metal phenoxyacetates were obtained using B3LYP/6-311++G ${ }^{* *}$ method. Geometric and magnetic aromaticity indices, dipole moments, and energies were calculated. Atomic charges on the atoms of phenoxyacetic acid molecule and its alkali metal salts were calculated by Mulliken, APT (atomic polar tensor), NPA (natural population analysis), MK (Merz-Singh-Kollman method), and ChelpG (charges from electrostatic potentials using grid-based method) methods. The theoretical wavenumbers and intensities of IR as well as chemical shifts in NMR spectra were obtained and compared with experimental data. The effect of alkali metals on molecular structure of phenoxyacetic acid appears in the shift of selected bands along the series of alkali metal salts. The correlations between chosen bands and some metal parameters, such as electronegativity, ionization energy, and atomic, and ionic radius, have been noticed.
\end{abstract}

Keywords: Alkali metal phenoxyacetates, FT-IR, NMR, UV, DFT, molecular structure

\section{Introduction}

Antimicrobial activity, anticancer, analgesic and anti-inflammatory are some of the important properties of phenoxyacetic acids, which are widely used as plant growth regulators and herbicides. Due to their solubility in water they can move in agricultural ecosystems and cause the pollution of surface and ground waters [1]. It seems to be interesting to study the formation of phenoxyacetate salts with common ions in soil. This paper is a continuation of our reports on the metal effect on the electronic system of carboxylic acids $[2,3]$. The influence of lithium, sodium, potassium, rubidium, and cesium on the electronic system of the salt molecules was analyzed in this paper.

\section{Experimental}

Lithium, sodium, potassium, rubidium, and cesium phenoxyacetates were prepared by dissolving the powder of phenoxyacetic acid in the water solution of the appropriate metal hydroxide in a stoichiometric ratio $(1: 1)$. All reagents were obtained from Aldrich Chemical Company. The solution was left at the room temperature for $24 \mathrm{~h}$ until the sample crystallized in the solid state. Precipitants were filtered, washed by water, and dried under reduced pressure at $110^{\circ} \mathrm{C}$. Obtained complexes were anhydrous-in the IR spectra of solid state samples the lack of bands characterized for crystallizing water was observed. 


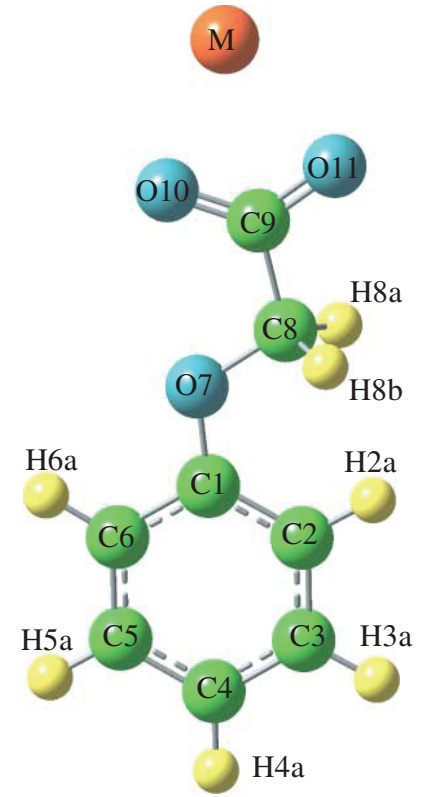

Figure 1: Atomic numbers in phenoxyacetate molecule.

The IR spectra were recorded with the Equinox 55, Bruker FT-IR spectrometer within the range $4000-400 \mathrm{~cm}^{-1}$. Samples in the solid state were measured in $\mathrm{KBr}$ pellets. The resolution of spectrometer was $1 \mathrm{~cm}^{-1}$. UV/Vis spectra were recorded with the DR/4000 U, HACH spectrophotometer. The NMR spectra of DMSO solution were recorded with the NMR AC 200 F Bruker unit. TMS was used as an internal reference.

The density functional (DFT) hybrid method B3LYP/6-311++G** was used to calculate optimized geometrical structures of studied compounds (Figure 1). All theoretical calculations were performed using the GAUSSIAN 09 [4] program running on a PC computer.

\section{Results and Discussion}

\subsection{Calculated Data}

Optimized geometrical structures of lithium (LiPA), sodium (NaPA), and potassium (KPA) phenoxyacetates were calculated using B3LYP/6-311++ $\mathrm{G}^{* *}$ method. The bond lengths and the angles between bonds in studied molecules are presented in Table 1 . The changes of almost all bond lengths in aromatic ring are noticed in the order from lithium to sodium phenoxyacetate molecules. The significant increasement of $\mathrm{C} 1-\mathrm{C} 2$ and $\mathrm{C} 6-\mathrm{C} 1$ bond lengths is observed in the series $\mathrm{LiPA}<\mathrm{NaPA}<\mathrm{KPA}$ (atomic numbers are shown in Figure 1). The bond lengths of $\mathrm{C} 3-\mathrm{C} 4$ and $\mathrm{C} 4-\mathrm{C} 5$ insignificantly increase whereas C2-C3 and C5-C6 insignificantly decrease in the above order. The significant decrease of C1-O7, C9O10, and C9-O11 bond lengths is also observed in the following order: LiPA $>$ NaPA $>$ KPA. But the significant increase is noticed for $\mathrm{O} 7-\mathrm{C} 8, \mathrm{C} 8-\mathrm{C} 9, \mathrm{O} 10-\mathrm{M}$, and $\mathrm{O} 11-\mathrm{M}$ bond lengths in the same series. As they might have been expected, the differences between $\mathrm{O} 10-\mathrm{M}$ and $\mathrm{O} 11-\mathrm{M}$ significantly decrease. 
Table 1: The bond lengths, angles, aromatic indices, values of dipole moment, and energy calculated (B3LYP/6-311++ $\mathrm{G}^{* *}$ ) for alkali metal phenoxyacetates (MPA).

\begin{tabular}{|c|c|c|c|}
\hline Atoms & LiPA & $\mathrm{NaPA}$ & KPA \\
\hline \multicolumn{4}{|c|}{ Bond lengths $(\AA)^{\mathrm{a}}$} \\
\hline $\mathrm{C} 1-\mathrm{C} 2$ & 1.3981 & 1.3992 & 1.3996 \\
\hline $\mathrm{C} 2-\mathrm{C} 3$ & 1.3978 & 1.3977 & 1.3976 \\
\hline C3-C4 & 1.3899 & 1.3901 & 1.3902 \\
\hline $\mathrm{C} 4-\mathrm{C} 5$ & 1.3982 & 1.3984 & 1.3985 \\
\hline C5-C6 & 1.3875 & 1.3873 & 1.3873 \\
\hline C6-C1 & 1.4013 & 1.4024 & 1.4029 \\
\hline $\mathrm{C} 1-\mathrm{O} 7$ & 1.3649 & 1.3617 & 1.3599 \\
\hline $\mathrm{O} 7-\mathrm{C} 8$ & 1.4121 & 1.4162 & 1.4183 \\
\hline C8-C9 & 1.5222 & 1.5290 & 1.5330 \\
\hline C9-O10 & 1.2601 & 1.2559 & 1.2544 \\
\hline C9-O11 & 1.2727 & 1.2689 & 1.2675 \\
\hline O10-M & 1.8691 & 2.2199 & 2.5286 \\
\hline O11-M & 1.8647 & 2.2169 & 2.5288 \\
\hline \multicolumn{4}{|c|}{ Angles $\left(^{\circ}\right)$} \\
\hline $\mathrm{C} 1-\mathrm{C} 2-\mathrm{C} 3$ & 119.48 & 119.55 & 119.58 \\
\hline $\mathrm{C} 2-\mathrm{C} 3-\mathrm{C} 4$ & 120.97 & 121.01 & 121.04 \\
\hline $\mathrm{C} 3-\mathrm{C} 4-\mathrm{C} 5$ & 119.13 & 119.07 & 119.05 \\
\hline $\mathrm{C} 4-\mathrm{C} 5-\mathrm{C} 6$ & 120.62 & 120.63 & 120.63 \\
\hline $\mathrm{C} 5-\mathrm{C} 6-\mathrm{C} 1$ & 120.03 & 120.14 & 120.20 \\
\hline $\mathrm{C} 6-\mathrm{C} 1-\mathrm{C} 2$ & 119.77 & 119.59 & 119.50 \\
\hline C6-C1-O7 & 115.45 & 115.55 & 115.61 \\
\hline $\mathrm{C} 2-\mathrm{C} 1-\mathrm{O} 7$ & 124.78 & 124.87 & 124.89 \\
\hline $\mathrm{C} 1-\mathrm{O} 7-\mathrm{C} 8$ & 118.63 & 118.64 & 118.70 \\
\hline O7-C8-C9 & 111.03 & 111.45 & 111.67 \\
\hline C8-C9-O10 & 121.99 & 120.84 & 120.52 \\
\hline C8-C9-O11 & 115.83 & 114.37 & 113.94 \\
\hline C9-O11-M & 82.55 & 87.23 & 90.75 \\
\hline O10-C9-O11 & 122.18 & 124.79 & 125.54 \\
\hline M-O10-C9 & 72.85 & 87.41 & 91.07 \\
\hline \multicolumn{4}{|c|}{ Aromaticity indices } \\
\hline HOMA $^{b}$ & 0.979262 & 0.976809 & 0.975763 \\
\hline$A_{J}^{\mathrm{c}}$ & 0.99714 & 0.99672 & 0.99656 \\
\hline $\mathrm{BAC}^{\mathrm{d}}$ & 0.92688 & 0.92338 & 0.92207 \\
\hline$I_{6}{ }^{\mathrm{e}}$ & 95.81867 & 95.52024 & 95.41015 \\
\hline $\mathrm{NICS}^{\mathrm{f}}$ & -9.1055 & -8.8957 & -8.9725 \\
\hline Dipole moment $(D)$ & 4.30 & 6.99 & 8.67 \\
\hline Energy $\left(\right.$ hartree) ${ }^{\mathrm{g}}$ & -542.55 & -697.26 & -1134.90 \\
\hline
\end{tabular}

${ }^{\mathrm{a}} 1 \AA=10^{-10} \mathrm{~m} ;{ }^{\mathrm{b}}$ abbreviation from harmonic oscillator model of aromaticity; ${ }^{\mathrm{c}}$ normalized function of variance of bond lengths; ${ }^{\mathrm{d}}$ bond alternation coefficient; ${ }^{\mathrm{e}}$ Bird's index [5]; ${ }^{\mathrm{f}}$ nuclear independent chemical shifts; ${ }^{\mathrm{g}} 1$ hartree $=2625.5 \mathrm{~kJ} / \mathrm{mol}$. 


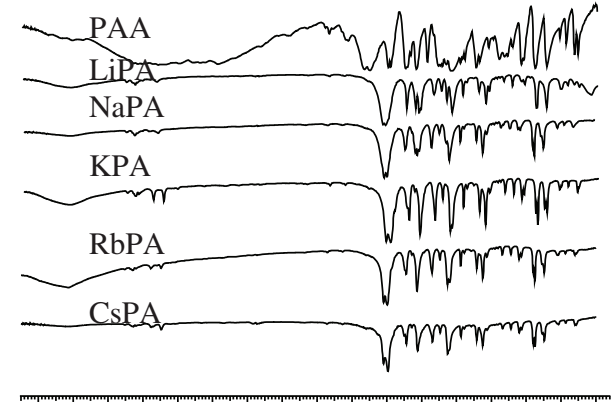

3600320028002400200016001200800400

Wavenumber $\left(\mathrm{cm}^{-1}\right)$

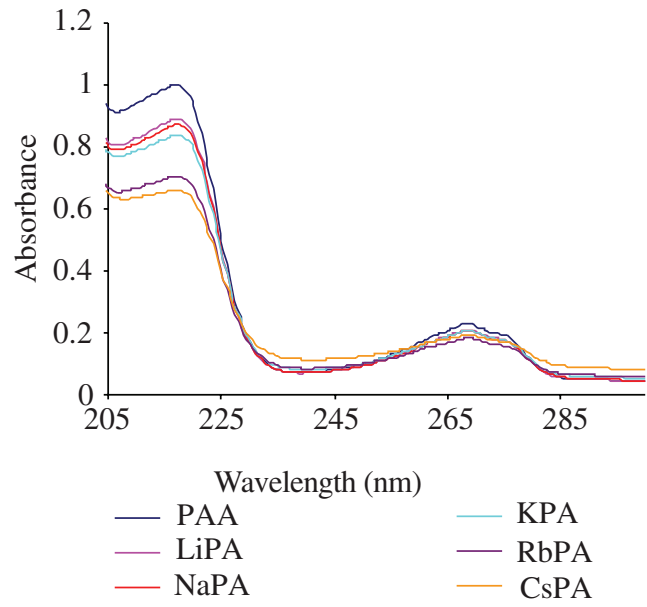

(b)

Figure 2: Experimental FT-IR spectra registered in $\mathrm{KBr}$ pellet (a) and UV spectra (b) for phenoxyacetic acid (PAA) and its salts (LiPA: lithium, NaPA: sodium, KPA: potassium, RbPA: rubidium, and CsPA: cesium phenoxyacetates).

In the case of angles, the increase in the order LiPA $<\mathrm{NaPA}<\mathrm{KPA}$ was observed for $\mathrm{C} 1-\mathrm{C} 2-\mathrm{C} 3$, $\mathrm{C} 2-\mathrm{C} 3-\mathrm{C} 4$, and $\mathrm{C} 5-\mathrm{C} 6-\mathrm{O} 1$ angles in the aromatic ring, but the decrease in this order was noticed for $\mathrm{C} 3-\mathrm{C} 4-\mathrm{C} 5$ and $\mathrm{C} 6-\mathrm{C} 1-\mathrm{C} 2$. The angles between carbon, oxygen, and metal atoms in alkil chain of molecules increase with exception of C8-C9-O10 and C8-C9-O11 angles.

Geometric and magnetic aromaticity indices [5-7], dipole moments, and energies were calculated and also shown in Table 1. Almost all aromaticity indices decrease in the following order: LiPA > $\mathrm{NaPA}>$ KPA. Values of energy also decrease in the same series. It might indicate the increase of stability of salt molecules in this order. Mulliken, APT, NPA, MK, and ChelpG atomic charges on the atoms of phenoxyacetic acid molecule and its alkali metal salts are gathered in Table 2. The increase of total charge of carboxylate group (negative values) are observed in the order LiPA $<\mathrm{NaPA}<\mathrm{KPA}$ when Mulliken and APT methods were used for calculation. In NPA method there are no changes whereas the decrease is observed for MK and ChelpG methods. The total charge in aromatic ring is not changed in above series irrespective of used method.

The theoretical wavenumbers of IR and Raman spectra as well as chemical shifts in NMR spectra were obtained and compared with experimental spectra.

\subsection{FT-IR, FT-Raman, and UV Spectra}

The vibrational (in $\mathrm{KBr}$ ) and electronic spectra of synthesized alkali metal phenoxyacetates were recorded and presented in Figure 2. In the UV spectra there were no changes in the wavelengths. The influence of studied metal cations on the vibrational structure of phenoxyacetates appears in the shift of selected bands along the metal series. The wavenumbers of asymmetric stretching vibration bands of carboxylate group in IR spectra decrease in the series LiPA $\rightarrow$ CsPA; however, for rubidium phenoxyacetate the insignificant increase is noted in comparison to potassium salt. In Raman spectra 


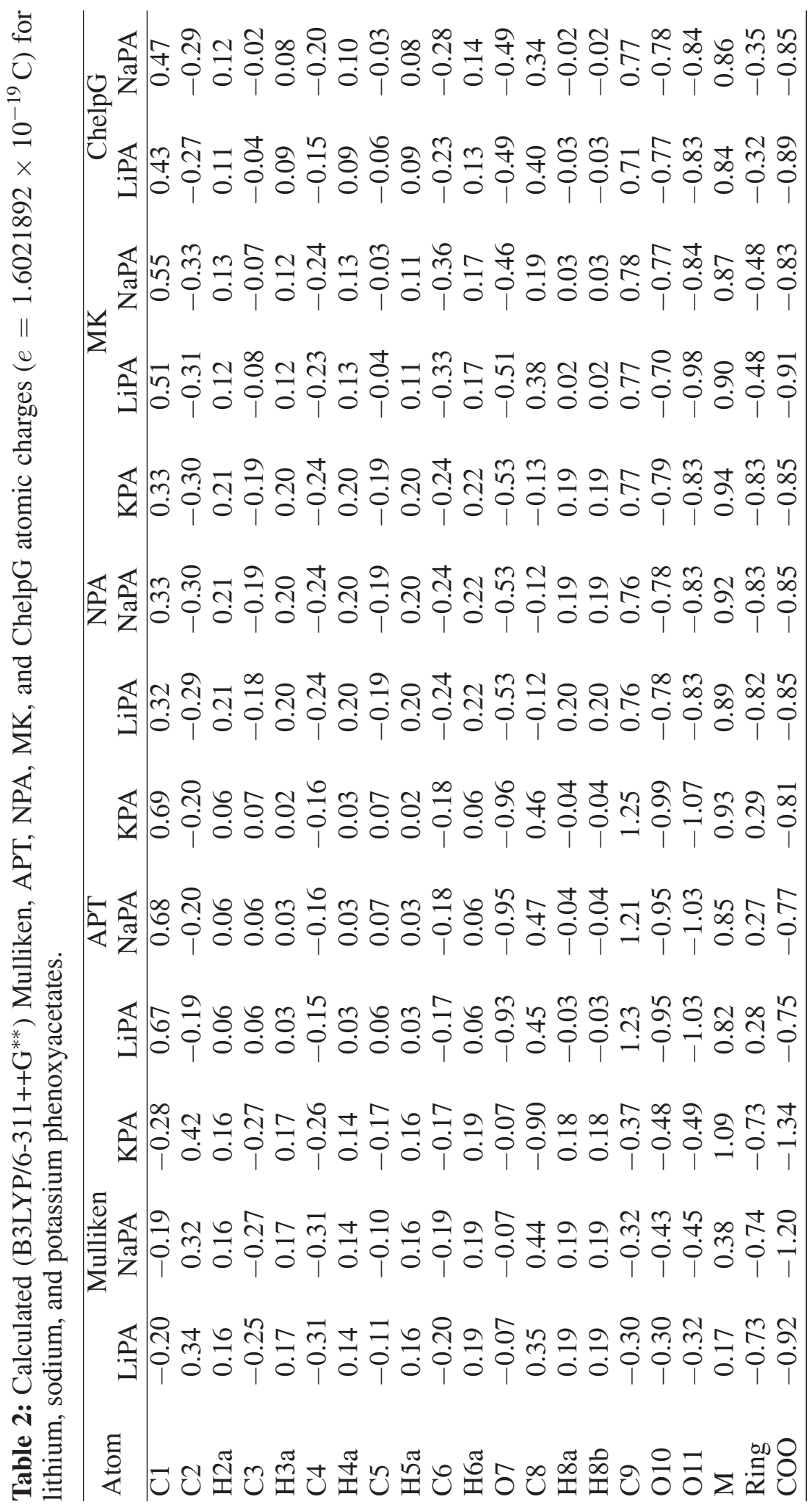


there are no changes in this series. For symmetric stretching vibration bands in Raman spectra the increase of the wavenumbers is noticed from lithium to cesium phenoxyacetate, but in IR spectra the wavenumbers increase to potassium phenoxyacetate and from KPA to CsPA the decrease is observed. The magnitudes of separation between wavenumbers due to asymmetric and symmetric stretching vibration bands $\Delta \nu_{\mathrm{as}-\mathrm{s}}=\nu_{\mathrm{as}}(\mathrm{COO})-\nu_{\mathrm{s}}(\mathrm{COO})$ in Raman spectra decrease in the series LiPA $>$ $\mathrm{NaPA}=\mathrm{KPA}>\mathrm{RbPA}>\mathrm{CsPA}$. The corresponding values for lithium, sodium, potassium, rubidium, and cesium phenoxyacetates in IR spectra are equal to 382, 361, 353, 357, and 361, respectively. The wavenumbers of asymmetric in-plane deformations $\beta_{\text {as }}(\mathrm{COO})$ decreased in the following series LiPA $>$ $\mathrm{NaPA}>\mathrm{KPA}>\mathrm{RbPA}>\mathrm{CsPA}$, whereas the magnitudes of separation between wavenumbers due to asymmetric and symmetric in plane deformations of $\mathrm{COO}^{-}$group increase in this order. The values of $\Delta \beta=\beta_{\mathrm{s}}(\mathrm{COO})-\beta_{\text {as }}(\mathrm{COO})$ are equal to $518,524,528,530$, and $536 \mathrm{~cm}^{-1}$. The wavenumbers as well as intensity of the aromatic ring bands did not significantly change.

The correlations between chosen bands and some metal parameters, such as electronegativity, ionization energy, atomic and ionic radius, atomic mass, and inverse of atomic mass [8] have been studied. The best correlation was obtained for ionic radius and ionization energy for band of $\nu(\mathrm{C}-\mathrm{O}-\mathrm{C})$ etheric group vibration $\left(R^{2}=0.978\right)$.

\subsection{NMR Spectra}

Values of the experimental as well as calculated (B3LYP/6-311++ $\mathrm{G}^{* *}$ ) chemical shifts in ${ }^{1} \mathrm{H}$ and ${ }^{13} \mathrm{C}$ NMR spectra of lithium, sodium, potassium, rubidium, and cesium phenoxyacetates are presented in Table 3. Data for the last two salts are only experimentally obtained. Experimental chemical shifts show decreasing tendency in the series $\mathrm{Li}>\mathrm{Na}>\mathrm{K}>\mathrm{Rb}>\mathrm{Cs}$ salts for H8a and H8b protons, but for protons connected with the aromatic ring the decrease was observed only for lithium, sodium, and potassium phenoxyacetates, and for rubidium and cesium salts these values increase in comparison to potassium one. The changes in ${ }^{13} \mathrm{C}$ NMR spectra indicated decreasing tendency from lithium to potassium phenoxyacetate, and then the increase from potassium to rubidium, and finally the insignificant decrease for cesium salt were observed for almost all carbon atoms in the aromatic ring (except of $\mathrm{C} 1$ and $\mathrm{C} 4$ ) and $\mathrm{C} 9$ atom. For $\mathrm{C} 1$ and $\mathrm{C} 8$ carbon atoms the opposite tendency was observed, whereas an insignificant decreasing tendency was observed for $\mathrm{C} 4$ carbon atom in the series LiPA $=\mathrm{NaPA}>\mathrm{KPA}>\mathrm{RbPA}>$ CsPA.

\section{Conclusions}

Some regular changes were observed in the calculated values of studied alkali metal phenoxyacetates. Almost all bond lengths decreased in the series LiPA $>$ NaPA $>$ KPA. All geometric aromaticity indices decreased in the same order. Values of energy also decreased in the studied series. On the other hand the shift of selected bands in IR, Raman, and NMR spectra along the phenoxyacetate series was also observed.

The calculated parameters were compared to the experimental characteristics of studied compounds. Good correlation between experimental and theoretical IR and Raman spectra was noted. The correlation coefficients $\left(R^{2}\right)$ for IR spectra for lithium, sodium, and potassium phenoxyacetates amount to $0.9986,0.9990$, and 0.9984 , respectively. The corresponding values for Raman spectra are 0.9970, 
Table 3: Calculated (B3LYP/6-311++G**) as well as experimental chemical shifts $\left({ }^{1} \mathrm{H}\right.$ and ${ }^{13} \mathrm{C}$ NMR) of alkali metal phenoxyacetates (ppm).

\begin{tabular}{lcccccccc}
\hline \multirow{2}{*}{ Atom } & \multicolumn{2}{c}{ LiPA } & \multicolumn{2}{c}{ NaPA } & \multicolumn{2}{c}{ KPA } & RbPA & CsPA \\
& Exp. & Calc. & Exp. & Calc. & Exp. & Calc. & Exp. & Exp. \\
\hline H2a & 6.848 & 6.640 & 6.834 & 6.650 & 6.781 & 6.660 & 6.828 & 6.825 \\
H3a & 7.211 & 7.283 & 7.204 & 7.297 & 7.185 & 7.269 & 7.194 & 7.196 \\
H4a & 6.835 & 6.889 & 6.818 & 6.923 & 6.760 & 6.880 & 6.786 & 6.787 \\
H5a & 7.211 & 7.293 & 7.204 & 7.321 & 7.185 & 7.301 & 7.194 & 7.196 \\
H6a & 6.848 & 7.274 & 6.834 & 7.209 & 6.781 & 7.209 & 6.828 & 6.825 \\
H8a & 4.210 & 4.029 & 4.110 & 4.237 & 4.010 & 4.121 & 4.080 & 4.060 \\
H8b & 4.210 & 4.093 & 4.110 & 4.236 & 4.010 & 4.121 & 4.080 & 4.060 \\
C1 & 158.76 & 166.94 & 159.01 & 166.11 & 159.30 & 166.48 & 159.08 & 159.18 \\
C2 & 129.06 & 113.06 & 128.93 & 112.4 & 128.88 & 112.26 & 128.96 & 128.92 \\
C3 & 119.80 & 133.33 & 119.50 & 133.58 & 119.15 & 133.55 & 119.40 & 119.27 \\
C4 & 114.50 & 122.86 & 114.50 & 123.02 & 114.41 & 122.53 & 114.40 & 114.39 \\
C5 & 119.80 & 134.07 & 119.50 & 133.6 & 119.15 & 133.39 & 119.40 & 119.27 \\
C6 & 129.06 & 123.04 & 128.93 & 122.74 & 128.88 & 122.78 & 128.96 & 128.92 \\
C8 & 67.48 & 68.15 & 67.83 & 67.58 & 68.11 & 67.72 & 67.61 & 67.96 \\
C9 & 172.75 & 178.09 & 170.85 & 184.5 & 169.25 & 184.05 & 169.62 & 169.53 \\
\hline
\end{tabular}

0.9975, and 0.9973. The linear correlation between calculated and experimental data of NMR spectra was also observed. Correlation coefficients for ${ }^{13} \mathrm{C}$ NMR are in the range $0.9002-0.9063$ and for ${ }^{1} \mathrm{H}$ NMR the range is $0.9845-0.9878$.

It was interesting to note the linear correlation between wavenumbers of $\nu_{\mathrm{s}}(\mathrm{COO})$ and $\nu_{\text {as }}(\mathrm{COO})$ bands and distances between $\mathrm{C} 9-\mathrm{O} 11$ and $\mathrm{O} 10-\mathrm{M}$. The corresponding correlation coefficients for $\nu_{\mathrm{s}}(\mathrm{COO})$ wavenumbers amount to 0.9951 and 0.9767 and for $\nu_{\mathrm{as}}(\mathrm{COO}) 0.9999$ and 0.9469 . Good correlation between chemical shifts in ${ }^{13} \mathrm{C}$ NMR spectra and Mulliken atomic charges on $\mathrm{C} 1$ and $\mathrm{C} 6$ atoms was also found $\left(R^{2}=0.9718\right.$ and 0.9932 , resp.).

\section{Acknowledgment}

The presented work was supported by Białystok University of Technology (Theme no. S/WBiIŚ/ 1/2012). 


\section{References}

[1] T. Cserháti and E. Forgács, "Phenoxyacetic acids: separation and quantitative determination," Journal of Chromatography B, vol. 717, no. 1-2, pp. 157-178, 1998.

[2] W. Lewandowski, M. Kalinowska, and H. Lewandowska, "The influence of halogens on the electronic system of biologically important ligands: spectroscopic study of halogenobenzoic acids, halogenobenzoates and 5-halogenouracils," Inorganica Chimica Acta, vol. 358, no. 7, pp. 2155-2166, 2005.

[3] M. Samsonowicz, R. Świsłocka, E. Regulska, and W. Lewandowski, "Comparison of molecular structure of alkali metal o-, m- and p-nitrobenzoates," Journal of Molecular Structure, vol. 887, no. 1-3, pp. 209-215, 2008.

[4] M. J. Frisch, G. W. Trucks, H. B. Schlegel et al., Gaussian 09 (Revision A.1), Gaussian, Wallingford, Conn, USA, 2009.

[5] T. M. Krygowski and M. Cyrański, "Separation of the energetic and geometric contributions to the aromaticity of $\pi$-electron carbocyclics," Tetrahedron, vol. 52, no. 5, pp. 1713-1722, 1996.

[6] P. Von Ragué Schleyer, M. Manoharan, Z. X. Wang et al., "Dissected nucleus-independent chemical shift analysis of $\pi$-aromaticity and antiaromaticity," Organic Letters, vol. 3, no. 16, pp. 2465-2468, 2001.

[7] R. Świsłocka, E. Regulska, M. Samsonowicz, and W. Lewandowski, "Experimental (FT-IR, FTRaman, 1H, 13C NMR) and theoretical study of alkali metal 2-aminonicotinates," Polyhedron, vol. 28, no. 16, pp. 3556-3564, 2009.

[8] J. Emsley, The Elements, Clarendon, Oxford, UK, 2nd edition, 1991. 


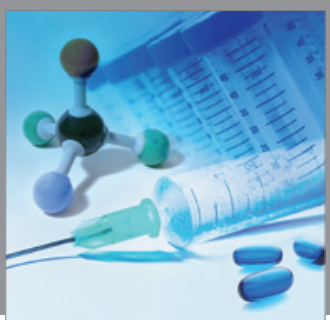

International Journal of

Medicinal Chemistry

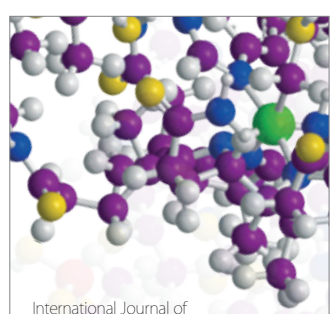

Carbohydrate Chemistry

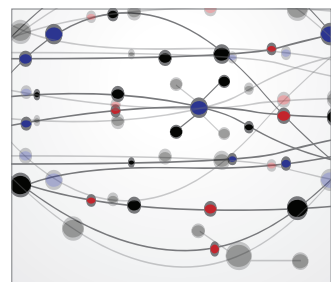

The Scientific World Journal
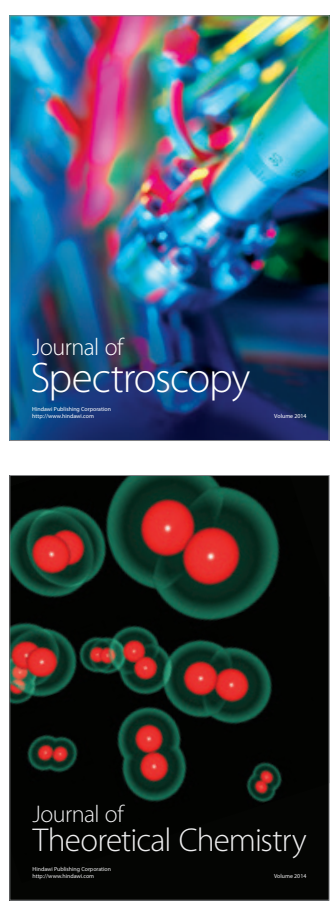
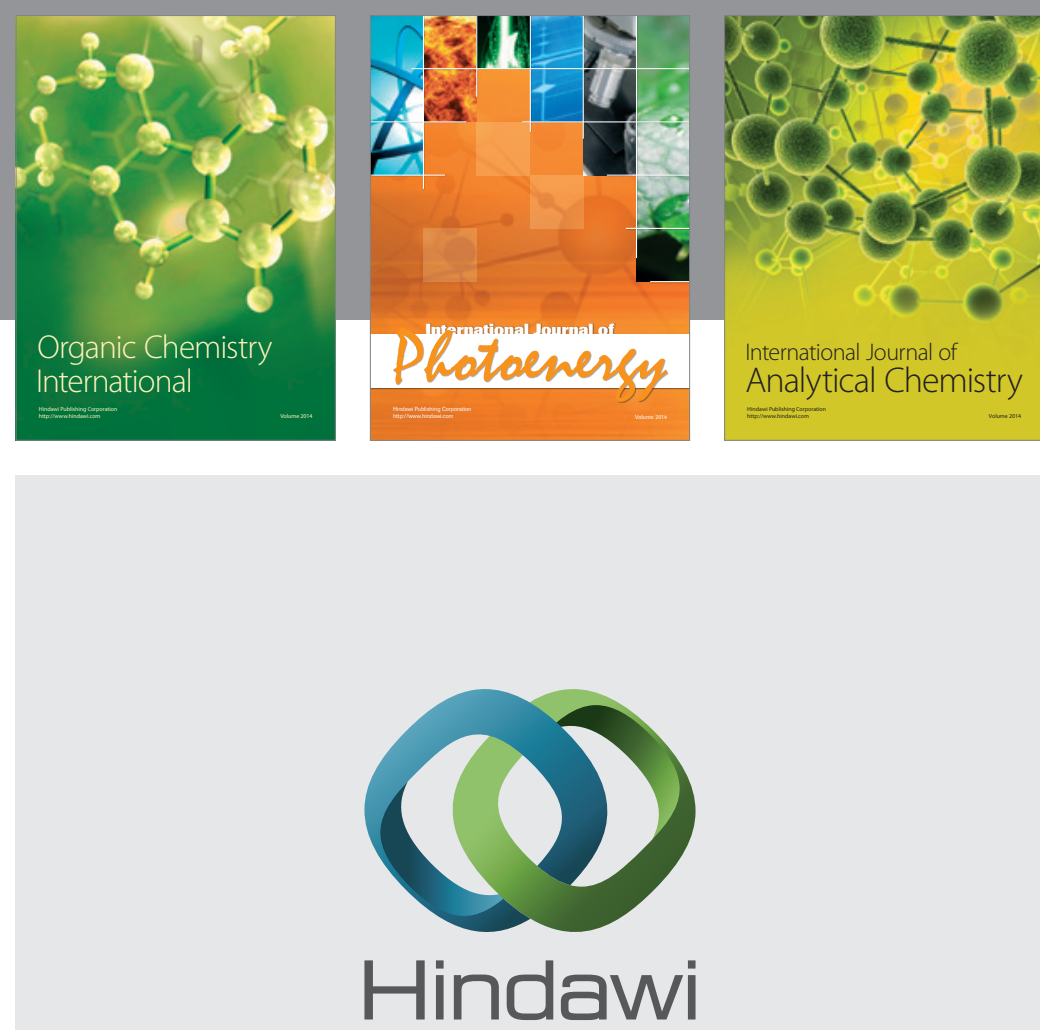

Submit your manuscripts at

http://www.hindawi.com
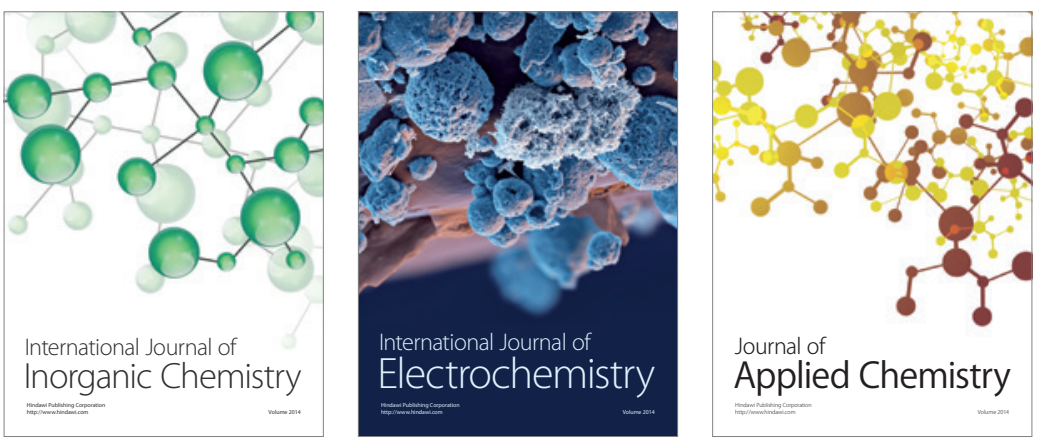

Journal of

Applied Chemistry
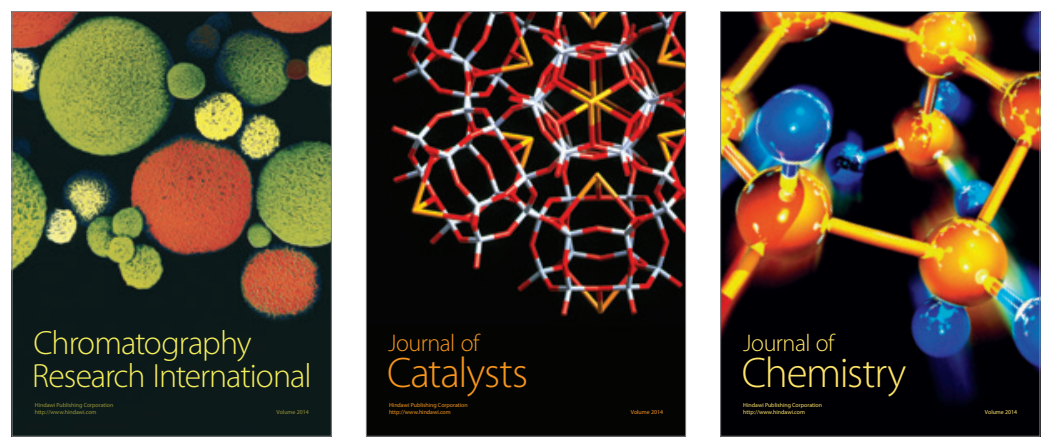
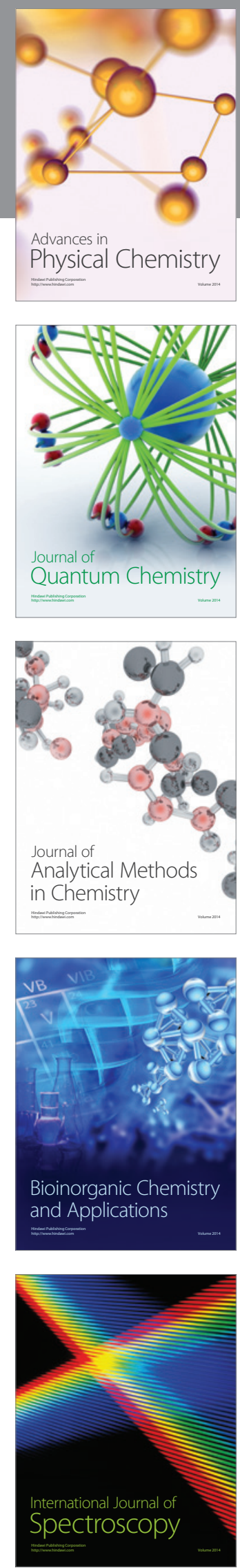